\title{
La estereotipia social de los adolescentes italianos durante la práctica deportiva The social stereotype of Italian adolescents during sports practice
}

*Eva Muñoz Jiménez, **Daniel Garrote Rojas, *Cristina Sánchez Romero, ***Stefano Martelli, ***Giovanna Russo *Universidad Nacional de Educación a Distancia (España), ${ }^{* *}$ Universidad de Granada (España), ***Università di Bologna (Italia)

\begin{abstract}
Resumen. El deporte es una de las actividades que más se realiza en el tiempo libre por los adolescentes, sin embargo los comportamientos estereotipados durante su práctica pueden influir en la actividad deportiva. El objetivo de esta investigación desarrollada en la ciudad de Bolonia (Italia), ha sido analizar los prejuicios y actitudes de los adolescentes que hacen deporte en su tiempo libre. Para ello, hemos aplicado el cuestionario «Sport e Integrazione sociale. Indagine sulle suole secundarie di secondo grado in Italia» (Caruso, G, et.al. 2018) a un grupo de adolescentes italianos $(\mathrm{N}=286)$ con edades comprendida entre los 12 y 15 años $(\mathrm{M}=12,72)$. Los resultados nos muestran diferencias significativas en sexo, discriminación y origen $(\mathrm{r}=.307)(\mathrm{Sig}=.000)$. Género y discriminación hacia la mujer $(\mathrm{r}=.133)$ $(\mathrm{Sig} .=.25)$; género y aspecto físico $(\mathrm{r}=.182)(\mathrm{Sig} .=.029) \mathrm{y}$, en mejorar tu aspecto físico $(\mathrm{M}=.416)(p=.012)$ frente a los hábitos de un grupo sedentario. En conclusión, todavía se confirman las imágenes estereotipadas a través del deporte respecto al género y al desarrollo de la actividad que se cuestionan continuamente.
\end{abstract}

Palabras clave: Adolescencia, deporte, exclusión social, prejuicios, inclusión.

Abstract. One of the activities that teenagers practice more often is sport in their free time, having said that stereotypical behaviours during their practice can influence sports activity. The aim of the present study has been to look into the different perceptions that adolescents have about social, ethnic, sports and gender stereotypes about prejudices and attitudes during sports activity as an inclusive environment; using the «Sport e Integrazione sociale. Indagine sulle suole secundarie di secondo grado in Italia» (Caruso, G, et.al. 2018) instrument. This study has been carried out in Bologna (Italy), with participants $(\mathrm{N}=286)$ between 12 and 15 years old $(\mathrm{M}=12.72)$. The results show that there are differences in gender, discrimination and origin $(\mathrm{r}=.307)(\mathrm{Sig}=.000)$; gender and discrimination towards women $(r=.133)($ Sig. $=.25)$; gender and physical appearance $(r=.182)($ Sig. $=.029)$; and improving your physical appearance $(M=$ .416) $(\mathrm{p}=.012)$ versus the habits of a sedentary group. In conclusion, the stereotyped images through sport regarding gender and the development of the activity that are continually questioned are still confirmed.

Keywords: Adolescence, sport, social exclusion, prejudice, inclusion.

\section{Introducción}

El objetivo de esta investigación ha sido analizar los prejuicios y actitudes de los adolescentes que hacen deporte en su tiempo libre. El ocio y el tiempo libre son fundamentales para el desarrollo humano.

Una de las actividades que caracteriza al ocio es, la práctica deportiva. A través de la práctica deportiva, se consiguen importantes beneficios para la salud, tanto a nivel físico como mental, por ejemplo, se ha visto que el nivel de autoconcepto de los adolescentes aumenta cuando los adolescentes realizan actividad deportiva de manera habitual (Adarve et al., 2019). También, mencionar la importancia de la inteligencia emocional para el bienestar psicológico y las relaciones positivas de los adolescentes, a partir de diferentes estudios, se subraya el beneficio de la actividad deportiva y el desarrollo y entrenamiento de manera transversal de las destrezas emocionales (Gallardo Peña et al., 2018; Acebbes Sánchez \& Rodríguez- Romo, 2019; Fernández-Espinosa \& Almagro, 2019)

Por otro lado, el deporte como entorno social desempeña un papel importante en la vida de muchos adolescentes (Malinauskas, Juodsnukis, 2017; Trottier, Robitaille, 2014). Se ha demostrado a través de varios estudios que el deporte tiene un efecto en la adquisición y desarrollo de habilidades sociales entre los adolescentes (Lemonia et al., 2017; Lintunen \& Gould, 2015; Vaquero-Solís et al., 2018).

Cuando los adolescentes son socialmente competentes, sus relaciones interpersonales son una fuente de bienestar $\mathrm{e}$

Fecha recepción: 31-05-20. Fecha de aceptación: 26-08-20

Cristina Sánchez Romero

csanchez@edu.uned.es identidad. Sin embargo, algunos adolescentes que son rechazados por otros pares, pudiendo enfrentarse a desafíos sociales, psicológicos y de salud mental que podrían tener efectos negativos en su bienestar. Para facilitar relaciones satisfactorias y alejadas de conductas inactivas y nocivas para la salud, conviene crear entornos que favorezcan las relaciones sociales satisfactorias (Lizandra \& Peiró-Velert, 2019), utilizando el deporte como herramienta educativa para el desarrollo de actitudes y valores positivos (Monjas et al., 2015; Rodríguez-Groba et al., 2017) y también, como herramienta de integración y desarrollo de las prácticas educativas inclusivas (Asís-Roig, 2017; Russo, 2019; Ramírez-Díaz \& Cabeza Ruíz, 2020). En esta línea de educación inclusiva, la práctica deportiva puede ser una estrategia de prevención contra conductas violentas o bullying en el aula (Medina Cascales, J\& Prieto, 2018).

Diversos estudios muestran que la práctica regular de deporte durante la niñez y la adolescencia contribuye a la adquisición de habilidades sociales como la cooperación, la búsqueda de derechos, la conciencia de las responsabilidades, el establecimiento de la empatía, el autocontrol y el liderazgo; como consecuencia, el nivel de agresividad disminuye y se mantienen relaciones positivas entre pares (PerronGoinas, Brendgen \& Vitaro, 2017; Shachar, et al. 2016; Calero et al., 2017; Inoue et al., 2018; Kavussanu \& Stanger, 2017). Además, las actividades deportivas animan a los niños a ayudar a los demás y a desarrollar altruismo y empatía (Lee et al., 2014). También, se ha observado que los adolescentes que practican deportes son más amigables con sus compañeros, especialmente con aquellos que participan en actividades similares, y tienen menos contacto con sus compañeros que están inclinados a participar en conductas antisociales (Jodra et al., 2018). 
Por ello, nos parece de gran importancia analizar la percepción de los adolescentes italianos durante el desarrollo de la actividad deportiva. De forma que se trabajen actitudes que eviten el estereotipo (social, étnico, deportivo y de género) en los adolescentes. Está demostrado que se ha analizado en este ámbito la existencia de la exclusión de algunos colectivos. En este sentido, existen diferentes programas europeos para analizar, trabajar y formar sobre los distintos tipos de discriminación, directa e indirecta, que pueden tener lugar en la práctica deportiva de los adolescentes (RodríguezGroba et al., 2017).

Respecto al género, destacamos la existencia de estudios sobre la discriminación sexual en la práctica del ejercicio físico entre los hombres y la mujeres preadolescente en el tiempo de ocio (Dosal Ulloa et al., 2017; Gil-Madrona et al., 2017; Sanz-Gil, 2017).

Esta investigación se ha desarrollado en Italia, en un Colegio público de Castesano. El Colegio estaba interesado en conocer las relaciones entre los alumnos/coetáneos en su ambiente extraescolar para así poder analizar los perjuicios y actitudes que surgen fuera del horario escolar y, de este modo, mejorar y trabajar desde el Centro diferentes programas educativos buscando una mejora en la convivencia.

Actualmente, Italia promueve un compromiso ético y educativo del deporte en todos los niveles, siendo el deporte una actividad de ocio relativamente joven, ya que desde los años' 90 del siglo pasado se promueve el compromiso ético y educativo del deporte, renovando el significado asociado con el fenómeno del deporte, apoyado en el pasaje:

- De un deporte competitivo a un deporte cooperativo.

- De un deporte exclusivo hasta un deporte inclusivo.

- De un deporte performativo a un deporte educativo.

A través de un concepto del deporte fuertemente inclusivo «Sport per tutti» ya que forma parte de las actividades prosociales «en la medida en que el ejercicio genera un entorno social más o menos estructurado, expresando al mismo tiempo un carácter relacional predominante» (Cei, 2001).

\section{Material y método}

\section{Metodología}

La investigación realizada se ha abordado desde una metodología de carácter cuantitativo. El diseño del estudio parte de un abordaje cuantitativo de tipo descriptivo, inferencial y ex post facto (Ruiz Olabuénaga, Villa, \& Álvarez, 2003) que nos ha permitido analizar los siguientes factores en los adolescentes italianos durante la actividad deportiva: género, edad, percepciones que tienen a cerca de estereotipia social, étnica, habito deportivo y género.

El objetivo de nuestro estudio fue analizar los perjuicios y actitudes que surgen durante la práctica deportiva en un conjunto de adolescentes italianos, analizando la existencia de diferencias entre el género (niños y niñas) en los diferentes ítems, mediante los análisis descriptivos, correlaciones y la prueba estadística T de Student en la línea de la investigaciones de (Flores, et al., 2013; Griffith, 2009; Rogerson, 2011)

El análisis de datos estadísticos se ha llevado a cabo utilizando el software del Programa de IBM Statistical Package para Ciencias Sociales (SPSS) versión 24.

\section{Instrumento}

El cuestionario que hemos utilizado es «Sport e Integrazione sociale. Indagine sulle scuole secundarie di secondo grado in Italia» (Caruso, Cerbara, Menneti, Misiti, \& Tintori, 2018) que nos permite analizar los objetivos planteados en nuestra investigación. Según los autores citados este cuestionario permite analizar entre otros los valores y significados atribuidos por los jóvenes a los estereotipos deportivos por el género y la étnica en la prácticas deportiva.

El cuestionario evalúa 5 secciones/dimensiones:

- Sección: Tus relaciones: esta dimensión se centra en conocer la relación que tienen los participantes con sus amigos y las actividades que realizan en el tiempo libre. Además de preguntar, la relación que tienen con la familia.

- Sección: Tu opinión: conocer la opinión que los sujetos tienen hacia ciertos problemas sociales.

- Sección: Deporte: conocimiento de la actividad física extraescolar que realizan los participantes.

- Sección: El deporte para ti es....: conocer la percepción que los participantes tiene a cerca de los valores que se transmiten a través de la práctica deportiva.

- Sección: Qué opinas sobre...: Esta dimensión se centra en los estereotipos y la categorización social a través del deporte.

A su vez este cuestionario nos permite analizar la práctica deportiva como proceso de inclusión. Con este cuestionario se puede analizar el deporte como factor de integración en las sociedades multiculturales» (Caruso, et al. 2018). El cuestionario tiene una consistencia interna utilizando el Alfa de Cronbach de 0,764.

\section{Participantes}

Esta investigación se ha desarrollado en Italia, la muestra seleccionada pertenece a un Colegio Público de Secundaria de I Grado de Castesano un municipio situado a 10 kilómetros de Bolonia (Provincia de Emilia-Romaña), con una población de 15.363 habitantes. El alumnado que acude al centro es de clase económica media.

El Centro consta de 18 clases con 455 estudiantes. La muestra de niños/as, fue seleccionada mediante un muestreo no probabilístico de tipo intencional, ya que nos permitió seleccionar aquellos casos accesibles que aceptaron ser incluidos en el estudio (Hernández, Fernández, y Baptista, 2014). La selección de la muestra no probabilística ha sido de 286 sujetos de los cuales son de género masculino $155(54,2 \%)$ y femenino 131 (45,8\%). Las características del alumnado se pueden ver en la Tabla 1 (Distribución por edad del alumnado) y Tabla 2 (Distribución del alumnado por curso). En cuanto a la nacionalidad de los participantes, el 95,8\% de los participantes es de nacionalidad italiana y el $3,8 \%$ proceden de otras nacionalidades.

\begin{tabular}{|c|c|c|c|}
\hline \multicolumn{4}{|c|}{ Distribución por edad del alumnado } \\
\hline 12 & 121 & 42,3 & 42,3 \\
\hline 13 & 124 & 43,4 & 85,7 \\
\hline 14 & 40 & 14,0 & 99,7 \\
\hline 15 & 1 &, 3 & 100 \\
\hline \multicolumn{4}{|c|}{$\begin{array}{l}\text { Tabla } 2 . \\
\text { Distribución del alumnado por curso }\end{array}$} \\
\hline Curso $\quad \mathrm{F}$ & Frecuencia & Porcentaje & Porcentaje acumulado \\
\hline Segundo & 145 & 50,7 & 50,7 \\
\hline Tercero & 141 & 49,3 & 100 \\
\hline
\end{tabular}




\section{Recolección de datos}

Para la recopilación de datos se pidió permiso al Centro, a los padres y a los participantes siguiendo las normas éticas para el desarrollo de la investigación con estudiantes (Consejo De Organizaciones Internacionales, Cioms, Oms, \& Who, 2002; Namakforoosh, 2000)

\section{Resultados}

El análisis de los resultados los hemos estructurado en las cinco secciones del cuestionario:

\section{Sección: Tus relaciones}

Las actividades que realizan con los participantes sus amigos en el tiempo libre, son las siguientes: Salen con ellos (plaza, centros comerciales, discotecas, etc.) el 26,9\% (44,3\% del sexo masculino y $42,9 \%$ del femenino); Hacen deporte/ actividad física juntos (al aire libre o en una estructura) el $24,7 \%$ (46,8\% del sexo masculino y $33,3 \%$ femenino); Se ven en una casa (videojuegos, Tv, Internet, chat, etc.) el 24,5\%, (44,7\% de los chicos y el 34,8\% de las chicas); Van a la parroquia, mezquita, iglesia el 5,3\% (9,9\% chicos y 7,4\% chicas); Quedan para estudiar el 28,7\% (34\% chicas y los chicos el 26,6\%).

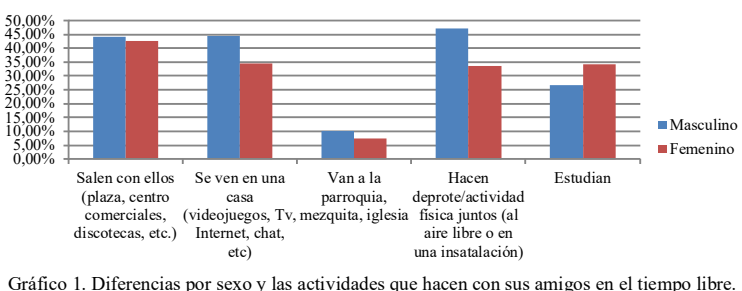

\section{Sección: Deporte}

En primer lugar, analizamos cuantos sujetos realizaban deporte fuera del horario escolar. En concreto, los participantes que realizaban actividad física en horario extraescolar eran de $\mathrm{N}=237(82,9 \%)$ y el grupo sedentario eran de $\mathrm{N}=40$ $(14 \%)$.

En cuanto al género, dentro del grupo masculino, realiza deporte $133(47,8 \%)$ y son sedentarios $17(6,1 \%)$ chicos. En cuanto al grupo femenino, realiza deporte $104(37,4 \%)$ y son sedentarias $23(8,3 \%)$ chicas.

\section{Hábitos del grupo activo}

La mayoría de los participantes llevan de 8 a 10 años practicando fuera del horario escolar $(34,6 \%)$, de 5 a 7 años $(24,8 \%)$ y hasta 4 años $(18,2 \%)$. En cuanto al género, tanto el grupo masculino $(22,8 \%)$ como el femenino $(16,1 \%)$ practican deporte desde hace 8 a 10 años. El 61,5\% de los sujetos realizan deporte de 2 a 3 veces por semana (39,5\% del grupo masculino y el $32,9 \%$ del grupo femenino), todos los días lo realizan el $13,6 \%$ y una vez a la semana $(9,8 \%)$. En cuanto al deporte que practican, el 20,3\% futbol, el 17,1\% baloncesto. Por género, los chicos practican más fútbol (el 21,6\% masculino) y baloncesto $(8,1 \%)$; y las chicas baloncesto $(10,8 \%)$ y danza $(8,5 \%)$.

El motivo por el cual los sujetos se motivan a realizar deporte, como primera opción, lo realizan por divertimento y pasión (31,2\%), adquirir habilidades nuevas $(15,3 \%)$, sentir- se bien, para desahogarse (14,8\%) y mejorar el aspecto físico $(12,4 \%)$. En cuanto a la diferencia por género, la mayoría de los sujetos realizan deporte por divertimento y pasión tanto en el grupo masculino (41,2\%) como el femenino (32,7\%). Posteriormente, el grupo masculino elige por adquirir habilidades y aprender cosas nuevas $(22,4 \%$ ) y el grupo femenino por estar bien y en forma $(19,2 \%)$.

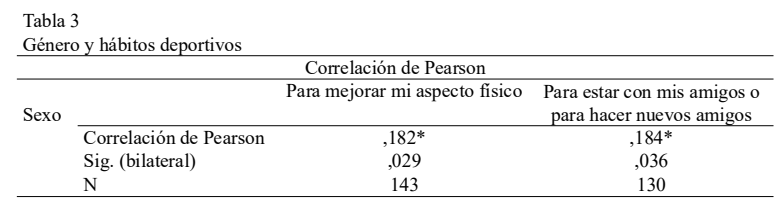

Existe una correlación significativa .01 (bilateral) entre las variables: Género y realizo deporte Para mejorar mi aspecto físico $(\mathrm{r}=.182)(\mathrm{Sig} .=.029)$. También existe una correlación significativa entre Sexo y realizo deporte Para estar con mis amigos o para hacer nuevos amigos ( $\mathrm{r}=.184)(\mathrm{Sig} .=.036)$.

\section{Hábitos del grupo sedentario}

En el grupo sedentario, hay un grupo que no realizan deporte actualmente, pero que alguna vez han realizado y lo han abandonado (14,7\%) y un grupo que nunca ha realizado deporte (2,1\%). En concreto la diferencia de sexos, dicen haber abandonado el deporte el grupo masculino (35,4\%) y el grupo femenino $(52,1 \%)$. Las razones principales de porque han abandonado el deporte son: No les gustó, se cansaron (40,3\%); el estudio (14,9\%); problemas con los compañeros con los que practicaba deporte, y cambio a otra ciudad (7,5\%). Sobre lo que piensan sus padres sobre el deporte: Es necesario para la salud (46\%), es algo que debo hace absolutamente $(27,6 \%)$, es útil aprender las reglas y respetar a todos (16,2\%), es una actividad costosa (2,3\%). En cuanto a la opinión de los participantes, los entrenadores y los formadores así como los aspectos «técnicos» del deporte deberían: Transmitir los valores positivos (29,9\%), hacer que los jóvenes hagan deporte (29,3\%), hay gente en la que puedas confiar (26,8\%), te animan a ganar a toda $\operatorname{costa}(9,6 \%)$.

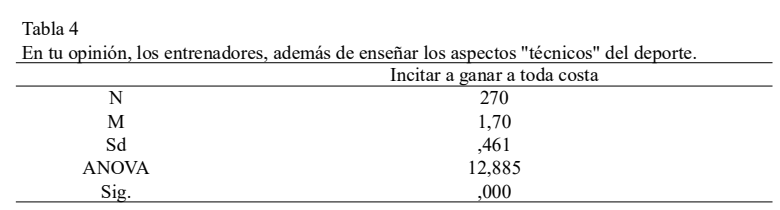

Como podemos observar, la diferencia de medias es significativa en la variable género y los entrenadores incitan a ganar a toda costa (F: 12,885; Sig.:.000).

\section{Sección: El deporte para ti es...}

La opinión que tienen los participantes sobre el deporte. Mayoritariamente opinan que con el deporte es posible, mejorar el aspecto físico, sentirse parte de un grupo, combatir tus miedos y aprender a resolver problemas.

Como podemos observar, la diferencia de medias es significativa en las variables:

- Género y Con el deporte es posible combatir tus miedos (F: 9,578; Sig.:.002).

- Género y Con el deporte es posible estar dispuesto a hacer cualquier cosa para ganar (F: 11,688; Sig...001). 


\begin{tabular}{|c|c|c|c|c|c|c|c|c|c|}
\hline & \multirow{2}{*}{$\begin{array}{c}\begin{array}{c}\text { Combatir } \\
\text { tus miedos }\end{array} \\
279\end{array}$} & \multirow{2}{*}{$\begin{array}{c}\begin{array}{c}\text { Mejorar tu } \\
\text { aspecto físico }\end{array} \\
280\end{array}$} & \multirow{2}{*}{$\begin{array}{c}\begin{array}{c}\text { Sentirse } \\
\text { excluido }\end{array} \\
279\end{array}$} & \multicolumn{3}{|c|}{$\begin{array}{c}\text { Aprende a resolver } \\
\text { problemas sin violencia }\end{array}$} & \multicolumn{3}{|c|}{$\begin{array}{l}\text { Sentirse parte } \\
\text { de un grupo }\end{array}$} \\
\hline $\mathrm{N}$ & & & & \multicolumn{3}{|c|}{279} & \multicolumn{3}{|c|}{282} \\
\hline M & & 1,06 & 1,85 & \multicolumn{3}{|c|}{1,40} & \multicolumn{2}{|c|}{1,09} & \\
\hline $\mathrm{Sd}$ & ,436 & ,239 &, 355 & \multicolumn{3}{|c|}{,491 } & \multicolumn{2}{|c|}{,280 } & \\
\hline ANOVA & 9,578 & , 173 & 7,154 & \multirow{2}{*}{\multicolumn{3}{|c|}{$\begin{array}{l}, 023 \\
, 880 \\
\end{array}$}} & \multirow{2}{*}{\multicolumn{2}{|c|}{$\begin{array}{c}1,719 \\
, 191 \\
\end{array}$}} & \\
\hline (Sig.) & ,002 & ,677 & ,008 & & & & & & \\
\hline \multicolumn{10}{|l|}{$\begin{array}{l}\text { Tabla } 6 \\
T \text { de Stud }\end{array}$} \\
\hline & 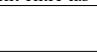 & (2) & tentas & Género & $\mathrm{N}$ & $\begin{array}{l}\text { Medias } \\
\text { por sexo }\end{array}$ & $\mathrm{T}$ & $P$ & $\mathrm{Df}$ \\
\hline \multicolumn{4}{|c|}{ Combatir tus miedos } & $\begin{array}{l}\text { Masculino } \\
\text { Femenino }\end{array}$ & $\begin{array}{l}0149 \\
130\end{array}$ & $\begin{array}{l}1,33 \\
1,17\end{array}$ & 3,095 & ,002 & 277 \\
\hline \multicolumn{4}{|c|}{ Mejorar tu aspecto físico } & Masculino & o 151 & 1,07 & 416 &, 012 & 278 \\
\hline \multicolumn{4}{|c|}{ Sentirse excluido } & $\begin{array}{l}\text { Masculino } \\
\text { Femenino }\end{array}$ & $\begin{array}{l}151 \\
\text { o } 151 \\
129\end{array}$ & 1,80 & $-2,675$ & ,008 & 277 \\
\hline \multicolumn{4}{|c|}{ Aprende a resolver problemas sin violencia } & $\begin{array}{l}\text { Masculino } \\
\text { Femenino }\end{array}$ & $\begin{array}{l}151 \\
128\end{array}$ & $\begin{array}{l}1,40 \\
1,41\end{array}$ &,- 151 &, 880 & 277 \\
\hline \multicolumn{4}{|c|}{ Sentirse parte de un grupo } & $\begin{array}{l}\text { Masculino } \\
\text { Femenino }\end{array}$ & $\begin{array}{l}\text { o } 152 \\
130\end{array}$ & $\begin{array}{l}1,11 \\
1,06\end{array}$ & 1,311 & ,191 & 280 \\
\hline \multicolumn{4}{|c|}{ Ser discriminada por ser mujer } & $\begin{array}{l}\text { Masculino } \\
\text { Femenino }\end{array}$ & $\begin{array}{l}\text { o } 149 \\
130\end{array}$ & $\begin{array}{l}1,93 \\
1,93\end{array}$ &,- 676 &, 500 & 277 \\
\hline \multicolumn{4}{|c|}{ Generar enfrentamientos y conflictos } & $\begin{array}{l}\text { Masculino } \\
\text { Femenino }\end{array}$ & $\begin{array}{l}\text { o } 150 \\
128\end{array}$ & $\begin{array}{l}1,71 \\
1,84\end{array}$ & $-2,559$ &, 011 & 276 \\
\hline \multicolumn{4}{|c|}{ Estar dispuesto a hacer cualquier cosa para ganar } & $\begin{array}{l}\text { Masculino } \\
\text { Femenino }\end{array}$ & $\begin{array}{l}\text { o } 151 \\
126\end{array}$ & $\begin{array}{l}1,46 \\
1,66\end{array}$ & $-3,419$ &, 001 & 275 \\
\hline \multicolumn{10}{|c|}{$\begin{array}{l}\text { Nota: } \mathrm{T}=\text { Estadística de prueba calculada, } \mathrm{P}=\text { Nivel de significancia, } \mathrm{Df}=\mathrm{Grados} \text { de libertad } \\
\text { Con respecto a la Tabla } 6 \text {, podemos ver las diferencias significativas entre las variables } \\
\text { independientes Género y Con el deporte es posible..., donde la media en el grupo masculino } \\
\text { tiene un promedio más alto en las variables Combatir tus miedos }(\mathrm{M}=1,33)(\mathrm{p}=, 002) \text {; Mejorar tu } \\
\text { aspecto fisico }(\mathrm{M}=.416)(\mathrm{p}=.012) \text {. El grupo femenino tiene un promedio más alto en las } \\
\text { variables Sentirse excluido }(\mathrm{M}=1,91)(\mathrm{p}=.008) \text {; Generar enfrentamientos y conflictos }(\mathrm{M}=1,84) \\
(\mathrm{p}=.011) \text {; Estar dispuesto a hacer cualquier cosas para ganar }(\mathrm{M}=1,66)(\mathrm{p}=.001) \text {. }\end{array}$} \\
\hline
\end{tabular}

\section{Sección: Con el deporte no es posible...}

Los participantes opinan que con el deporte no es posible, sentirse excluido, ser excluido por ser de sexo femenino y generar confrontamientos y conflictos. En cuanto a lo que enseña el deporte, los sujetos están muy de acuerdo en que enseña comprometerse a obtener resultados $(61,9 \%)$; aprender de las derrotas (54,2\%); el respeto por las reglas (47,2\%); y el respeto a los adversarios (42\%).

Tabla 7

\begin{tabular}{|c|c|c|c|c|c|c|}
\hline 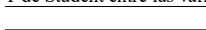 & Género & $\mathrm{N}^{\circ}$ & Medias por género & $\mathrm{t}$ & $P$ & Df \\
\hline \multirow[t]{2}{*}{ Respeto a las reglas } & Masculino & 150 & 4,05 &,- 111 & 912 & 277 \\
\hline & Femenino & 129 & 4,06 & & & \\
\hline \multirow[t]{2}{*}{ Luchar por los resultados } & Masculino & 152 & 4,51 & 1,413 & ,159 & 274 \\
\hline & Femenino & 129 & 4,36 & & & \\
\hline Respeto a los oponentes & $\begin{array}{l}\text { Masculino } \\
\text { Femenino }\end{array}$ & $\begin{array}{l}151 \\
130\end{array}$ & $\begin{array}{l}3,98 \\
3,85\end{array}$ & ,928 &, 354 & 276 \\
\hline Aprender de las derrotas. & $\begin{array}{l}\text { Masculino } \\
\text { Femenino }\end{array}$ & $\begin{array}{l}150 \\
129\end{array}$ & $\begin{array}{l}4,02 \\
4,31\end{array}$ & $-2,050$ & ,041 & 277 \\
\hline
\end{tabular}

\section{Sección 5- Qué opinas sobre...}

Los participantes opinan que no están para nada de acuerdo, con que para animar a tu equipo, la violencia es aceptable $(72,4 \%)$ y algunos deportes no son adecuados para mujeres $(49,7 \%)$.

\begin{tabular}{lcccc}
$\begin{array}{l}\text { Tabla } 8 \\
\text { Qué opinas sobre: }\end{array}$ & \multicolumn{4}{l}{} \\
\hline $\begin{array}{c}\text { Es mucho mejor } \\
\text { tener un entrenador } \\
\text { masculino }\end{array}$ & $\begin{array}{c}\text { Algunos deportes no Para practicar deportes } \\
\text { son adecuados para } \\
\text { mujeres }\end{array}$ & $\begin{array}{c}\text { Para animar a tu } \\
\text { debs tener } \\
\text { fisico }\end{array}$ & $\begin{array}{c}\text { equipo, la violencia } \\
\text { es aceptable }\end{array}$ \\
\hline N & 279 & 277 & 278 & 275 \\
M & 2,80 & 3,16 & 2,97 & 3,60 \\
Sd & 1,032 & 1,022 & 1,012 &, 811 \\
ANOVA & 28,257 & 74,730 & 32,988 & 10,738 \\
(Sig.) &, 000 &, 000 &, 000 &, 001 \\
\hline
\end{tabular}

Se observa que, la diferencia de medias es significativa en las variables:

- Género y Es mucho mejor tener un entrenador masculino (F: 28,257; Sig.:.000).

- Género y Algunos deportes no son adecuados para mujeres (F: 74,730; Sig.:.000).

- Género y Para practicar deporte debes tener físico (F:32,988; Sig.:.,001).

\begin{tabular}{ccccc} 
criminada & $\begin{array}{c}\text { Generar enfrentamientos y } \\
\text { conflictos }\end{array}$ & $\begin{array}{c}\text { Estar dispuesto a hacer } \\
\text { cualquier cosa para ganar }\end{array}$ & $\begin{array}{c}\text { Combatir tus } \\
\text { miedos }\end{array}$ & $\begin{array}{c}\text { Estar dispuesto a hacer } \\
\text { cualquier cosa para ganar }\end{array}$ \\
\hline 279 & 278 & 277 & 279 & 277 \\
, 94 & 1,77 & 1,55 & 1,25 & 1,55 \\
246 &, 424 &, 499 &, 436 &, 499 \\
457 & 6,548 & 11,688 & 9,578 & 11,688 \\
500 &, 011 &, 001 &, 002 &, 001 \\
\hline
\end{tabular}

- Género y Para animar a tu equipo, la violencia es aceptable (F: 10,738; Sig.:.001).

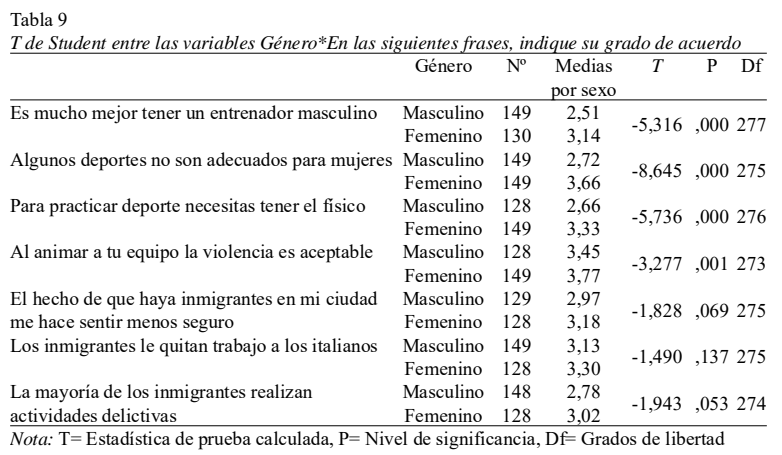

La Tabla 9 muestra las diferencias significativas entre las variables Género y El grado de acuerdo en las siguientes frases. A continuación, se muestra las medias en las cuales el grupo femenino tiene un promedio más alto que el grupo masculino: Es mucho mejor tener un entrenador masculino $(\mathrm{M}=3,14)(p=, 000)$; Algunos deportes no son adecuados para mujeres $(\mathrm{M}=3,66)(\mathrm{p}=, 000)$; Para practicar deporte necesitas tener físico $(3,33)(p=, 000)$; Al animar a tu equipo la violencia es aceptable $(\mathrm{M}=3,77)(p=, 001)$. El valor $\mathrm{p}$ es en estos casos inferior a $0,05 \mathrm{y}$, por lo tanto, se considera significativo desde un punto de vista estadístico.

\section{Discusión}

La sociedad está preocupada por la utilización que del tiempo libre hacen los más jóvenes. La Organización Mundial de la Salud (OMS) alerta de que el 80\% de los adolescentes no realiza el ejercicio físico recomendado siendo el deporte fundamental para el desarrollo humano. El deporte además de ser una actividad profesional, se considera una actividad lúdica definida como aquello que se puede realizar en el tiempo libre, con el objetivo de liberar tensiones, huir de la rutina diaria y de las preocupaciones, y para obtener un poco de placer, diversión y entretenimiento (UNICEF-ONUUNESCO, 2004).

Los conceptos de tiempo libre y deporte para todos han transformado el modelo deportivo, haciendo que, la manifestación más habitual del deporte, la competitiva, pase en estos momentos a un segundo plano y sea sobre todo el deporte salud, recreación, la práctica físico-deportiva en espacios no convencionales o en la misma naturaleza. Mencionaremos, por ello, que en los últimos años, ha aumentado el interés en la relación entre la exposición a espacios verdes y la salud mental de niños y adolescentes. La evidencia sugiere constantemente una asociación beneficiosa entre la exposición a espacios verdes y las dificultades emocionales y de comportamiento de los adolescentes.

Como hemos visto en nuestro estudio, la frecuencia de los deportes y la participación de individuos en actividades deportivas está influenciada en una cierta proporción por 
factores ambientales como el lugar para vivir, las políticas que se enfocan en la inclusión social y por características demográficas como género, edad, estado civil y nivel socioeconómico (Hatfield et al., 2015; Lozano-Zapata et al., 2018; Oliviera-Brochado et al., 2017).

Por otro lado, los factores que motivan a las personas a participar en el deporte son: un estilo de vida saludable, estar en forma y la socialización (Oliveira - Brochado et al., 2017). Por lo tanto, el deporte se presenta como una herramienta clave para la inclusión social de los adolescentes y brinda la oportunidad a los adolescentes de alcanzar el capital social Bailey (2005). Cuando los adolescentes son socialmente competentes, sus relaciones interpersonales son una fuente de bienestar e identidad. Los adolescentes que practican deportes mejoran sus habilidades sociales y la integración social durante su práctica (Russo, 2019).

Con respecto a los estereotipos y la categorización social a través del deporte. En el estudio llevado a cabo por Ramírez-Díaz \& Cabeza-Ruiz (2020) para conocer la actitud de alumnado de Educación Secundaria hacia la diversidad sexual en el ámbito deportivo, se puede advertir que una parte del alumnado presenta actitudes negativas hacia la diversidad sexual, por lo que se considera necesario plantear líneas de actuación educativas que trabajen contra esta problemática.

Por lo que el deporte, también se ha convertido en una herramienta de inclusión (Gil-Madrona et al., 2017; MuñozJiménez et al., 2017; Segura et al, 2013). Debemos tener en cuenta que, los deportes que involucran compartir el mismo entorno con otras personas o que involucran trabajo en equipo requieren habilidades sociales como la internalización de normas sociales, la organización de comportamientos según las reglas, el respeto a los demás, la comunicación con otros, la cooperación con el grupo, la responsabilidad y proporcionar comentarios apropiados (Gould et al.,2012).

\section{Conclusión}

El deporte se presenta como una herramienta socializadora en la línea de estudios Brustad (2016). El 24,7\% de los participantes hacen deporte/actividad física con sus amigos en el tiempo libre, (al aire libre o en una estructura) (46,8\% del sexo masculino y $33,3 \%$ femenino).

En la sección deporte, un grupo de participantes afirman que realiza deporte fuera de su horario escolar $(82,9 \%)$, y otro grupo es sedentario (14\%). En cuanto al género, los chicos practican fútbol y baloncesto y las chicas baloncesto y baile. Siendo el motivo por el cual practican deporte por diversión, adquirir habilidades nuevas, sentirse bien y mejorar el aspecto físico. El grupo sedentario dice que alguna vez ha realizado deporte y que lo ha abandonado porque no les gustó, por el estudio o porque no hay instalaciones cerca de casa. Aunque todavía se confirman las imágenes estereotipadas a través del deporte respecto al género y se cuestionan continuamente como afirman Elling y Knoppers (2005).

Los participantes opinan que con el deporte no es posible, sentirse excluido, ser excluido por ser de sexo femenino y generar confrontamientos y conflictos. En cuanto a lo que enseña el deporte, los sujetos están muy de acuerdo en que enseña comprometerse a obtener resultados, aprender de las derrotas, el respeto por las reglas y el respeto a los adversarios.

Además, los participantes no están de acuerdo en que para animar a tu equipo, la violencia es aceptable y que algunos deportes no sean adecuados para mujeres.

Como conclusión diremos, que los participantes de este estudio, opinan que en el deporte es posible mejorar el aspecto físico, sentirse parte de un grupo, combatir el miedo y aprender a resolver problemas. Y no es posible sentirse excluido, crear confrontamiento y conflicto, ser excluido por ser de sexo femenino. Y que a través del deporte se aprende a comprometerse a obtener resultados, aprender de las derrotas, el respeto de las reglas y el respeto a los oponentes. En la sección qué opinas, los sujetos no están nada de acuerdo en que en el ánimo de su propio equipo, la violencia sea aceptable.

\section{Referencias}

Acebes Sánchez, J., \& Rodríguez-Romo, G. (2019). Relaciones entre el nivel de actividad física, experiencia deportiva e inteligencia emocional en alumnos de Grado de la Comunidad Madrid: Estudio de protocolo (Relation between physical activity levels, sport experience, and emotional intelligence among u. Retos, 36(36), 297-301. Recuperado de: https://doi.org/10.47197/ retos.v36i36.68970

Adarve, M., Zurita Ortega, F., Gómez Sánchez, V., Padial Ruz, R., \& Lara Sánchez, A. (2019). Influencia de la práctica de actividad física en el autoconcepto de adolescentes (Influence of the practice of physical activity on the self-concept of adolescents). Retos, 36(36), 342-347. Recuperado de: https:// doi.org/10.47197/retos.v36i36.68852

Asís-Roig, R. de. (2017). Reflexiones sobre discapacidad, deporte e inclusión $=$ Reflections on Disability, Sport and Inclusion. UNIVERSITAS. Revista de Filosofía, Derecho y Política, 27, 8. Recuperado de: https://doi.org/10.20318/universitas.2018.4016

Bailey, R. (2005). Evaluating the relationship between physical education, sport and social inclusión. Educational Review, 57(1), 71-90. doi:10.1080/0013191042000274196

Brochado, A., Brito, P., \& Oliveira-Brochado, F. (2017). Correlates of adults' participation in sport and frequency of sport. Science \& Sports. 32. Recuperado de: https://doi.org/10.1016/ j.scispo.2017.03.005

Brustad, R. J. (2016). Integrating Socialization Influences into the Study of Ciildren's Motivation in Sport. Journal of Sport and Exercise Psychology. Recuperado de: https://doi.org/10.1123/ jsep.14.1.59

Calero, A. D., Barreyro, J. P., \& Injoque-Ricle, I. (2017). Inteligencia emocional durante la adolescencia: su relación con la participación en actividades extracurriculares. Psicodebate. Recuerado de: https://doi.org/10.18682/pd.v17i2.693

Caruso, M.G., Cerbara, L., Menniti, A. \& Tintori, A. (2018). Sport e integrazione sociale. Istituto di Ricerche sulla Populazione e le Politiche Sociali (IRPPS). WP108

Cei, A. (2001). Sport per tutti: Sport a misura di ciascuno. In Psicologia della Salute (Vol. 2001, Issue 3). Recuperado de: https://doi.org/ $10.1400 / 63945$

CONSEJO DE ORGANIZACIONES INTERNACIONALES DE LAS CIENCIASMÉDICAS (CIOMS). Pautas éticas internacionales para la investigación biomédica en seres humanos. CIOMS, Ginebra 2002. Edición en español por el Programa Regional de Bioética OPS/OMS, Santiago de Chile, 2003, 118 pp.

Dosal Ulloa, R., Mejía Ciro, M. P., \& Capdevila Ortis, L. (2017). Deporte y equidad de género. Economía UNAM, 14(40), 121133. Recuperado de: https://doi.org/10.1016/ j.eunam.2017.01.005

Elling, A., \& Knoppers, A. (2005). Sport, gender and ethnicity: 
Practises of symbolic inclusion/exclusion. Journal of Youth and Adolescence. Recuperado de: https://doi.org/10.1007/s10964005-4311-6

Fernández-Espínola, C., \& Almagro, B. J. (2019). Relación entre motivación e inteligencia emocional en Educación Física: una revisión sistemática (Relation between motivation and emotional intelligence in Physical Education: A systematic review). Retos, 36(36), 584-589. Recuperado de: https:/doi.org/ 10.47197/retos.v36i36.64968

Flores, et al. (2013). Prueba t de Student. Inst Mex Seguro Soc, 51(3), 300-303

Gallardo Peña, M. A., Domíngez Escribano, M., \& González González de Mesa, C. (2018). Inteligencia emocional y conducta agresiva en el deporte ¿Puede inferir la modalidad deportiva y las horas de entrenamiento? (Emotional intelligence and aggressive behavior in sport. Can sports modality and hours of training infer?). Retos, (35), 176-180. Recuperado de: https://doi.org/10.47197/ retos.v0i35.66213

Gil-Madrona, P., Valdivia-Moral, P., González-Víllora, S., \& ZagalazSánchez, M. L. (2017). Percepciones y comportamientos de discriminación sexual en la práctica de ejercicio físico entre los hombres y mujeres preadolescentes en el tiempo de ocio. Revista de Psicologia Del Deporte.

Gould, D., Flett, R., \& Lauer, L. (2012). The relationship between psychosocial developmental and the sports climate experienced by underserved youth. Psychology of Sport and Exercise. 13 80-87. Recuperado de: https://doi.org/10.1016/ j.psychsport.2011.07.005

Griffith, A. (2009). SPSS For Dummies (2nd Edition). In 2nd Edition. Recuperado de: https://doi.org/10.1080/10436929908580238

Hatfield, D. P., Chomitz, V. R., Chui, K. K., Sacheck, J. M., \& Economos, C. D. (2015). Demographic, physiologic, and psychosocial correlates of physical activity in structured exercise and sports among low-income, overweight children. Journal of Nutrition Education and Behavior, 47(5), 452-458. Recuperado de: https://doi.org/10.1016/j.jneb.2015.05.008

Hernández-Sampieri, R., Fernández-Collado, C., \& Baptista-Lucio, M. (2014). Selección de la muestra. Metodología de La Investigación. (6 ${ }^{\mathrm{a}}$ ed., pp. 170-191). México: McGraw-Hill

Inoue, Y., Heffernan, C., Yamaguchi, T., \& Filo, K. (2018). Social and charitable impacts of a charity-affiliated sport event: A mixed methods study. Sport Management Review, 21(2), 202-218. Recuperado de: https://doi.org/10.1016/j.smr.2017.06.005

Jodra, P., Domínguez, R., \& Maté-Muñoz, J. L. (2018). Incidencia de la práctica deportiva en la conducta disruptiva de niños y adolescentes. Ágora Para La Educación Física y El Deporte. Recuperado de: https://doi.org/10.24197/aefd.2-3.2017.193-206

Kavussanu, M., \& Stanger, N. (2017). Moral behavior in sport. In Current Opinion in Psychology (Vol. 16, pp. 185-192). Elsevier B.V. Recuperado de: https://doi.org/10.1016/j.copsyc.2017.05.010

Lee, K. U., Kim, H. R., \& Yi, E. S. (2014). The effect of push factors in the leisure sports participation of the retired elderly on resocialization recovery resilience. Journal of Exercise Rehabilitation, 10(2), 92-99. Recuperado de: https://doi.org/ 10.12965/2Fjer. 140090

Lemonia, D., Goulimaris, D., \& Georgios, M. (2017). Social skills and prediction of the quality of life of adolescents. The case of dance and physical activities. Journal of Physical Education and Sport. Recuperado de: https://doi.org/10.7752/jpes.2017.s2076.

Lintunen, T., \& Gould, D. (2015). Developing social and emotional skills. In Routledge Comaninon to Sport and Exercise Psychology. Recuperado de: https://doi.org/10.4324/9781315880198.ch40

Lizandra, J., \& Peiró-Velert, C. (2019). Las relaciones sociales y su papel en la motivación hacia la práctica de actividad física en adolescentes: Un enfoque cualitativo (Social relatedness and its role in adolescents' motivation towards physical activity: a qualitative approach). Retos, 37(37), 41-47. Recuperado de: https://doi.org/10.47197/retos.v37i37.70374

Lozano-Zapata, R. E., Castrillón-Jaimes, Y. C., Peña-Reyes, M. S., \& Bustos-Viviescas, B. J. (2018). El esfuerzo físico y la salud en actividades recreativas y deportivas. Respuestas, 23(S1), 105108. Recuperado de: https://doi.org/10.22463/0122820x.1528
Malinauskas, R.K., \& Juodsnukis, D.R. (2017). Education of social responsibility among sports schools students. European Journal of Contemporary Education, 6(2), 289-296.

Medina Cascales, J., \& Prieto, M. J. (2018). Incidencia de la práctica de actividad física y deportiva como reguladora de la violencia escolar (Incidence of the practice of physical and sporting activities as a regulator of school violence). Retos, (35), 54-60. Recuperado de: https://doi.org/10.47197/retos.v0i35.64359

Monjas Aguado, R., Ponce Garzarán, A., \& Gea Fernández, J. M. (2015). La transmisión de valores a través del deporte. Deporte escolar y deporte federado: relaciones, puentes y posibles transferencias. Retos. Nuevas tendencias en Educación Física, Ocio y Recreación, 28, 276-284.

Muñoz-Jiménez, E. M., Garrote,Rojas, D., \& Sánchez-Romero, C. (2017). La práctica deportiva en personas con discapacidad: motivación personal, inclusión y salud. International Journal of Developmental and Educational Psychology, 4(1), 145-152. Recuperado de: http://www.infad.eu/RevistaINFAD/OJS/index.php/ IJODAEP/article/view/1037

Namakforoosh, M. N. (2000). Metodología de la investigación. México: Limusa

Oliveira-Brochado, A., Brito, P. Q., \& Oliveira-Brochado, F. (2017). Correlates of adults' participation in sport and frequency of sport. Science and Sports. Doi: j.scispo.2017.03.005.

Perron-Gélinas, A., Brendgen, M. \& Vitaro, F. (2017). Can sports mitigate the effects of depression and aggression on peer rejection? Journal of Applied Developmental Psychology, 50, 26-35. Recuperado de: https://doi.org/10.1016/ j.appdev.2017.03.006.

Ramírez-Díaz, A., \& Cabeza-Ruiz, R. (2020). Actitudes hacia la diversidad sexual en el deporte en estudiantes de educación secundaria (Secondary Education Students' Attitudes toward Sexual Diversity in Sport). Retos, 38(38), 654-660. Recuperado de: https://recyt.fecyt.es/index.php/retos/article/view/77934

Rodríguez-Groba, A., Eirín-Nemiña, R. \& Alonso-Ferreiro, A. (2017). Materiales y recursos didácticos contra la discriminación y la exclusión en el deporte en edad escolar. Educatio Siglo XXI, 35(3 Noviembr), 85. Recuperado de: https://doi.org/10.6018/j/ 308911

Rogerson, P. (2011). Statistical Methods for Geography. In Statistical Methods for Geography. Recuperado de: https://doi.org/10.4135/ 9781849209953

Ruiz Olabuénaga, J. I., Villa, A., \& Álvarez, M. (2003). Técnicas de triangulación y control de calidad en la investigación socioeducativa. In Proyecto de calidad integrado.

Russo, G. (2019). Integration by Sport Activities: Resource or Only a Paradox. «Journal of Mediterranean Knowledge 4, 17-29.

Sanz-Gil, J. (2017). Una aproximación a las dificultades del deporte femenino en la alta competición: Hacia la equidad e igualdad. Acciónmotriz, 18, 29-36.

Segura, J., Martínez-Ferrer, J. O., Guerra, M., \& Barnet, S. (2013). Creencias sobre la inclusión social y el deporte adaptado de deportistas, técnicos y gestores de federaciones deportivas de deportes para personas con discapacidad. Revista Iberoamericana de Psicologia Del Ejercicio Y El Deporte, 8(1), 120-144.

Shachar, K., Ronen-Rosenbaum, T., Rosenbaum, M., Orkibi, H., \& Hamama, L. (2016). Reducing child aggression through sports intervention: The role of self-control skills and emotions. Children and Youth Services Review, 71, 241-249. Recuperado de: https://doi.org/10.2478/v10237-011-0031-x

Trottier, C., \& Robitaille, S. (2014). Fostering life skills development in high school and community sport: A comparative analysis of the coach's role. Sport Psychologist, 28, 10-21.

UNICEF-ONU-UNESCO. (2004). Deporte. recreación y juego. Unicef. Recuperado de: https://doi.org/10.1007/s13398-0140173-7.2

Vaquero-Solís, M., Amado-Alonso, D., Sánchez-Oliva, D., SánchezMiguel, P., \& Iglesias-Gallego, D. (2018). Inteligencia emocional en la adolescencia: motivación y actividad física. Revista Internacional de Medicina y Ciencias de La Actividad Física y Del Deporte, X, 1-14. Recuperado de: http://cdeporte.rediris.es/revista/inpress/artinteligencia1127.pdf 